\title{
ON CERTAIN CONVOLUTION INEQUALITIES
}

\author{
LARS INGE HEDBERG
}

\begin{abstract}
It is proved that certain convolution inequalities are easy consequences of the Hardy-Littlewood-Wiener maximal theorem. These inequalities include the Hardy-Littlewood-Sobolev inequality for fractional integrals, its extension by Trudinger, and an interpolation inequality by Adams and Meyers. We also improve a recent extension of Trudinger's inequality due to Strichartz.
\end{abstract}

The purpose of this note is to point out that certain convolution inequalities are easy consequences of the Hardy-Littlewood-Wiener maximal theorem. These inequalities include the Hardy-Littlewood-Sobolev inequality for fractional integrals, its extension by Trudinger [11], and an interpolation inequality by Adams and Meyers ([1], [1a]). We also improve a recent extension of Trudinger's inequality due to Strichartz [10].

Let $f$ be real-valued, Lebesgue measurable, and defined in $\boldsymbol{R}^{d}$. For $0<p<\infty$ we write $\|f\|_{p}=\left\{\int_{R^{d}}|f(x)|^{p} d x\right\}^{1 / p}$. For $0<\alpha<d$ the Riesz potentials $I_{\alpha}(f)$ are defined by $I_{\alpha}(f)(x)=\int_{R^{d}} f(y)|x-y| \alpha^{-d} d y$. The maximal function $M(f)$ is defined by $M(f)(x)=\sup _{r>0} r^{-d} \int_{|y|<r}|f(x+y)| d y$. We will denote various constants, independent of $f$, by $A$.

By the Hardy-Littlewood-Wiener maximal theorem, a simple proof of which is given by Stein $[9, \mathrm{I} .1]$,

$$
\|M(f)\|_{p} \leqq A\|f\|_{p}, \quad p>1 .
$$

If $f$ is supported by a finite ball $B$, then (see $[9$, I.5.2])

$$
\int_{B ;} M(f) d x \leqq A \int_{B^{3}}\left(1+|f| \log ^{+}|f|\right) d x .
$$

The following theorem is due to Hardy and Littlewood [3] for $d=1$, and to Sobolev [8] in the general case. A simple proof is given in [9, V.1.2].

THEOREM 1. Let $0<\alpha<d, 1<p<q<\infty$, and $1 / q=1 / p-\alpha / d$. Then $\left\|I_{\alpha}(f)\right\|_{q} \leqq A\|f\|_{p}$. If $f$ is supported by a ball $B$, and $1 / q=1-\alpha / d$, then $I_{\alpha}(f) \in L^{q}(B)$ if $j_{B}|f| \log ^{+}|f| d x<\infty$.

We first prove a simple lemma.

Received by the editors February 18, 1972 and, in revised form, May 2, 1972.

AMS 1970 subject classifications. Primary 26A33, 46E30, 46E35. 
Lemma. (a) If $0<\alpha<d$, then for all $x \in \boldsymbol{R}^{d}$ and $\delta>0$

$$
\int_{|y-x| \leqq \delta}|f(y)||x-y|^{\alpha-\alpha} d y \leqq A \delta^{\alpha} M(f)(x) .
$$

(b) If $\beta>0$, then

$$
\int_{|y-x| \geqq \delta}|f(y)||x-y|^{-\beta-d} d y \leqq A \delta^{-\beta} M(f)(x) .
$$

Proof. We only prove (a), the proof of (b) being similar. For any $x \in \boldsymbol{R}^{d}$ and any $\delta>0$

$$
\begin{aligned}
\int_{|y-x| \leqq \delta}|f(y)||x-y|^{\alpha-\alpha} d y & =\sum_{n=0}^{\infty} \int_{\delta 2^{-n-1}<|y-x| \leqq \delta 2^{-n}}|f(y)||x-y|^{\alpha-\alpha} d y \\
& \leqq A \sum_{n=0}^{\infty}\left(\delta 2^{-n}\right)^{\alpha}\left(\delta 2^{-n}\right)^{-\alpha} \int_{|y-x| \leqq \delta 2^{-n}}|f(y)| d y \\
& \leqq A \delta^{\alpha} M(f)(x) \sum_{n=0}^{\infty} 2^{-n \alpha}
\end{aligned}
$$

which proves (a).

Proof of Theorem 1. Let $p \geqq 1,0<\alpha<d$, and let $x \in \boldsymbol{R}^{d}$ and $\delta>0$ be arbitrary. By Hölder's inequality for $p>1$, and immediately for $p=1$,

$$
\int_{|y-x|>\delta}|f(y)||x-y|^{\alpha-d} d y \leqq A\|f\|_{p} \delta^{\alpha-d / p} .
$$

Thus, by the lemma

$$
\left|I_{\alpha}(f)(x)\right| \leqq A\left(\delta^{\alpha} M(f)(x)+\delta^{\alpha-\alpha / p}\|f\|_{p}\right) .
$$

To minimize this expression we choose $\delta=\left(M(f)(x) /\|f\|_{p}\right)^{-p / d}$. This gives

$$
\left|I_{\alpha}(f)(x)\right| \leqq A M(f)(x)^{1-\alpha p / d}\|f\|_{p}^{\alpha p / d}
$$

The theorem follows immediately from (1) and (2).

REMARK. The main difference between the above proof and the proof given in [9] is that the latter depends on the nondiagonal case of the Marcinkiewicz interpolation theorem, whereas the proof of the maximal theorem, as given e.g. in [9], only depends on the easier interpolation along the diagonal, and a covering lemma. On the other hand the proof in [9] is valid for more general kernels.

THEOREM 2. Suppose $f \in L^{p}\left(\boldsymbol{R}^{d}\right)$ has support in a ball $B$ with diameter $R$, and let $p=d / \alpha>1$. Then, for any $\varepsilon>0$ there exists a constant $A_{\varepsilon}$, 
independent of $f$ and $R$, such that

$$
\int_{B} \exp \left\{\frac{d}{\omega_{d-1}}\left|\frac{\left|I_{\alpha}(f)(x)\right|}{\|f\|_{p}}-\varepsilon\right|^{p /(p-1)}\right\} d x \leqq A_{\varepsilon} R^{d} .
$$

Here $\omega_{d-1}$ is the area of the $d-1$ dimensional unit sphere.

REMARK. The above inequality clearly implies that for any $\beta<d / \omega_{d-1}$ there is an $A$ so that

$$
\int_{r^{\prime}} \exp \beta\left(\left|I_{\alpha}(f)(x)\right| /\|f\|_{p}\right)^{p /(p-1)} d x \leqq A R^{d} .
$$

For $\alpha=1$ this implies the inequality of Trudinger [11, p. 479]. In fact, if $\varphi$ belongs to the Sobolev space $W_{1}^{\circ}(B)$, then $|\varphi| \leqq \omega_{d-1}^{-1} I_{1}(|\operatorname{grad} \varphi|)$. (See e.g. [9, V.2.2].) It is known ([4], [6]) that for $\alpha=1$ the inequality does not hold for $\beta>d / \omega_{d-1}$, but Moser [6] has proved that in this case the inequality is true for $\beta=d / \omega_{d-1}$, i.e. $\varepsilon$ above can be taken to be zero. The extension of Trudinger's inequality to $\alpha \neq 1$ is due to Strichartz [10], whose proof is also very simple, but does not seem to give quite as sharp a result.

Proof. Without loss of generality we can assume that $\|f\|_{p}=1$. As in the proof of Theorem 1 we obtain for any $x \in B$ and any $\delta, 0<\delta \leqq R$,

$$
\left|I_{\alpha}(f)(x)\right| \leqq A \delta^{\alpha} M(f)(x)+\left(\omega_{d-1} \log (R / \delta)\right)^{1-1 / p}
$$

Now choose $\delta^{\alpha}=\min \left\{\varepsilon A^{-1} M(f)(x)^{-1}, R^{\alpha}\right\}$. This gives

$$
\begin{gathered}
\left|I_{\alpha}(f)(x)\right| \leqq \varepsilon+\left(\omega_{d-1} \log ^{+}\left(R \varepsilon^{-1 / \alpha} A^{1 / \alpha} M(f)(x)^{1 / \alpha}\right)\right)^{1-1 / p} \\
\left(\left|I_{\alpha}(f)(x)\right|-\varepsilon\right)_{+}^{p /(p-1)} \leqq \omega_{d-1} d^{-1} \log ^{+}\left(R^{d} \varepsilon^{-p} A^{p} M(f)(x)^{p}\right)
\end{gathered}
$$

and the theorem follows.

The following theorem is a special case of Theorem 4 below, but we prove it separately because of the simplicity of the proof.

THEOREM 3. Let $f \geqq 0$ be measurable on $\boldsymbol{R}^{d}$. Then

$$
\left\|I_{\alpha \theta}(f)\right\|_{r} \leqq A\|f\|_{p}^{1-\theta}\left\|I_{\alpha}(f)\right\|_{a}^{\theta}
$$

with $0<\alpha<d, 0<\theta<1,1<p<\infty, p<q \leqq \infty$, and $1 / r=(1-\theta) / p+\theta / q$.

REMARK. For periodic functions, and without the restriction to positive functions, the theorem was proved by Hirschman [5]. For integral $\alpha$ and $\alpha \theta$ the theorem follows from the well-known inequalities of Gagliardo [2] and Nirenberg [7]. See also Theorem 4 below.

Proof. Let $x$ be arbitrary and let $\delta>0$. Then clearly

$$
\begin{aligned}
\int_{|y-x| \geqq \delta} f(y)|x-y|^{\alpha \theta-d} d y & \leqq \delta^{\alpha(\theta-1)} \int_{|y-x| \geqq \delta} f(y)|x-y|^{\alpha-\alpha} d y \\
& \leqq \delta^{\alpha(\theta-1)} I_{\alpha}(f(x)) .
\end{aligned}
$$


Thus, by the lemma $I_{\alpha \theta}(f)(x) \leqq A\left(\delta^{\alpha \theta} M(f)(x)+\delta^{\alpha(\theta-1)} I_{\alpha}(f)(x)\right)$. Choose $\delta^{\alpha}=I_{\alpha}(f)(x) / M(f)(x)$. It follows that

$$
I_{\alpha \theta}(f)(x) \leqq A M(f)(x)^{1-\theta} I_{\alpha}(f)(x)^{\theta} .
$$

The theorem follows immediately from Hölder's inequality and (1).

The following theorem was recently announced by Adams and Meyers [1]. Their proof, which is by complex interpolation, will appear in [1a].

THEOREM 4. Let $f \geqq 0$ be measurable on $\boldsymbol{R}^{d}$. Then

$$
\left\|I_{\alpha \theta}\left(f^{t}\right)\right\|_{r} \leqq A\|f\|_{p}^{t-\theta}\left\|I_{\alpha}(f)\right\|_{a}^{\theta},
$$

with $0<\alpha<d, 0<\theta<1,0<p<\infty, 0<q \leqq \infty, \theta<t<\theta+(1-\theta) p$, and $1 / r=(t-\theta) / p+\theta / q$.

Proof. The case $t=1$ was treated above. We first assume $t>1$. Then, by the assumptions, $t<p$. As before, by the lemma,

$$
\int_{|y-x| \leqq \delta} f^{t}(y)|x-y|^{\alpha \theta-d} d y \leqq A \delta^{\alpha \theta} M\left(f^{t}\right)(x) .
$$

Now choose $s<p$ so that $t<\theta+(1-\theta) s$. By Hölder's inequality

$$
\begin{aligned}
& \int_{|y-x| \geqq \delta} f^{t}(y)|x-y|^{\alpha \theta-d} d y \\
& \leqq\left\{\int_{|y-x| \geqq \delta} f(y)|x-y|^{\alpha-d} d y\right\}^{(s-t) /(s-1)} \\
& \cdot\left\{\int_{|y-x| \geq \delta} f^{s}(y)|x-y|^{-d-\alpha \eta /(t-1)} d y\right\}^{(t-1) /(s-1)},
\end{aligned}
$$

where $\eta=\theta-t+(1-\theta) s>0$.

By part (b) of the lemma

$$
\int_{|y-x| \geqq \delta} f^{s}(y)|x-y|^{-d-\alpha \eta /(t-1)} d y \leqq A \delta^{-\alpha \eta /(t-1)} M\left(f^{s}\right)(x) .
$$

We now observe that $M\left(f^{t}\right)^{1 / t} \leqq A M\left(f^{s}\right)^{1 / s}$ for $0<t \leqq s$. In fact for all $a>0$, by Hölder's inequality,

$$
\left\{a^{-d} \int_{|y-x|<a} f^{t}(y) d y\right\}^{1 / t} \leqq A\left\{a^{-d} \int_{|y-x|<a} f^{s}(y) d y\right\}^{1 / s} \leqq A M\left(f^{s}\right)(x)^{1 / s} .
$$

Thus

$I_{\alpha \theta}\left(f^{t}\right)(x) \leqq A \delta^{\alpha \theta} M\left(f^{s}\right)(x)^{t / s}+A \delta^{-\alpha \eta /(s-1)} M\left(f^{s}\right)(x)^{(t-1) /(s-1)} I_{\alpha}(f)(x)^{(s-t) /(s-1)}$. 
To minimize this expression we choose $\delta^{x}=I_{\alpha}(f)(x) / M\left(f^{s}\right)(x)^{1 / s}$. It follows that

$$
I_{\alpha \theta}\left(f^{t}\right)(x) \leqq A M\left(f^{s}\right)(x)^{(t-\theta) / s} I_{\alpha}(f)(x)^{\theta} .
$$

Since $\left\|M\left(f^{s}\right)^{1 / s}\right\|_{p} \leqq A\|f\|_{p}$ by (1), the theorem follows by Hölder's inequality.

Now assume $t<1$. We choose $s_{1}$ and $s_{2}$, so that $0<s_{2}<s_{1}<p, s_{1} \leqq t$, and so that $\theta+(1-\theta) s_{2}<t<\theta+(1-0) s_{1}$. By the assumptions this is possible.

Applying Hölder's inequality as in (6) we find

$$
\begin{aligned}
\int_{|y-x| \leqq \delta} f^{t}(y)|x-y|^{x \theta-d} d y & \leqq\left\{\int_{|y-x| \leqslant \delta} f(y)|x-y|^{\alpha-d} d y\right\}^{\left(t-s_{1}\right) /\left(1-s_{1}\right)} \\
& \cdot\left\{\int_{|y-x| \leqslant \delta} f^{s_{1}}(y)|x-y|^{-d+x \eta_{1} /(1-t)} d y\right\}^{(1-t) /\left(1-s_{1}\right)},
\end{aligned}
$$

where $\eta_{1}=\theta-t+(1-\theta) s_{1}>0$.

Similarly

$$
\begin{aligned}
\int_{|y-x| \geq \delta} f^{t}(y)|x-y|^{\alpha \theta-d} d y & \leqq\left\{\int_{|y-x| \geqq \delta} f(y)|x-y|^{\alpha-d} d y\right\}^{\left(t-s_{2}\right) /\left(1-s_{2}\right)} \\
& \cdot\left\{\int_{|y-x| \geqslant \delta} f^{s_{2}}(y)|x-y|^{-d+\alpha \eta_{2} /(1-t)} d y\right\}^{(1-t) /\left(1-s_{2}\right)},
\end{aligned}
$$

where $\eta_{2}=\theta-t+(1-\theta) s_{2}<0$.

By the lemma

$$
\int_{|y-x| \leqq \delta} f^{s_{1}}(y)|x-y|^{-d+x \eta_{1} /(1-t)} d y \leqq A \delta^{\alpha \eta_{1} /(1-t)} M\left(f^{s_{1}}\right)(x),
$$

and

$$
\begin{aligned}
\int_{|y-x| \geq \delta} f^{s_{2}}(y)|x-y|^{-d+\alpha \eta_{2} /(1-t)} d y & \leqq A \delta^{\alpha \eta_{2} /(1-t)} M\left(f^{s_{2}}\right)(x) \\
& \leqq A \delta^{\alpha \eta_{2} /(1-t)} M\left(f^{s_{1}}\right)(x)^{s_{2} / \varepsilon_{\sharp}}
\end{aligned}
$$

Thus

$$
\begin{aligned}
I_{\alpha \theta}\left(f^{t}\right)(x) \leqq & A \delta^{\alpha \eta_{1} /\left(1-s_{1}\right)} M\left(f^{s_{1}}\right)(x)^{(1-t) /\left(1-s_{1}\right)} I_{\alpha}(f)(x)^{\left(t-s_{1}\right) /\left(1-s_{1}\right)} \\
& +A \delta^{\alpha \eta_{2} /\left(1-s_{2}\right)} M\left(f^{s_{1}}\right)(x)^{s_{2}(1-t) / s_{1}\left(1-s_{2}\right)} I_{\alpha}(f)(x)^{\left(t-s_{2}\right) /\left(1-s_{2}\right)} \\
= & A \delta^{\alpha \theta}\left\{\delta^{\alpha\left(s_{1}-t\right)} M\left(f^{s_{1}}\right)(x)^{1-t} I_{\alpha}(f)(x)^{t-s_{1}}\right\}^{1 /\left(1-s_{1}\right)} \\
& +A \delta^{\alpha \theta}\left\{\delta^{\alpha\left(s_{2}-t\right)} M\left(f^{s_{1}}\right)(x)^{s_{2}(1-t) / s_{1}} I_{\alpha}(f)(x)^{t-s_{2}}\right\}^{1 /\left(1-s_{2}\right)} .
\end{aligned}
$$

By choosing $\delta^{\alpha}=I_{\alpha}(f)(x) / M\left(f^{s_{1}}\right)(x)^{1 / s_{1}}$, we find

$$
I_{\alpha \theta}\left(f^{t}(x)\right) \leqq A M\left(f^{s_{1}}\right)(x)^{(t-\theta) / s_{1}} I_{\alpha}(f)(x)^{\theta},
$$

which proves the theorem. 
Note that in the case $t<p, t<1$, we can simplify the proof by choosing $s_{1}=t$.

\section{REFERENCES}

1. D. R. Adams and N. G. Meyers, Bessel potentials. Inclusion relations among classes of exceptional sets, Bull. Amer. Math. Soc. 77 (1971), 968-970.

1a. - Bessel potentials. Inclusion relations among classes of exceptional sets (to appear).

2. E. Gagliardo, Ulteriori proprietà di alcune classi di funzioni in più variabili, Ricerche Mat. 8 (1959), 24-51. MR 22 \#181.

3. G. H. Hardy and J. E. Littlewood, Some properties of fractional integrals. I, Math. Z. 27 (1928), 565-606.

4. J. A. Hempel, G. R. Morris and N. S. Trudinger, On the sharpness of a limiting case of the Sobolev imbedding theorem, Bull. Austral. Math. Soc. 3 (1970), 369-373.

5. I. I. Hirschman, Jr., A convexity theorem for certain groups of transformations, J. Analyse Math. 2 (1953), 209-218. MR 15, 295; 1139.

6. J. Moser, $A$ sharp form of an inequality by $N$. Trudinger, Indiana Univ. Math. J. 20 (1971), 1077-1092.

7. L. Nirenberg, On elliptic partial differential equations, Ann. Scuola Norm. Sup. Pisa (3) 13 (1959), 115-162. MR 22 \#823.

8. S. L. Sobolev, On a theorem of functional analysis, Mat. Sb. 4 (46) (1938), 471-497; English transl., Amer. Math. Soc. Transl. (2) 34 (1963), 39-68.

9. E. M. Stein, Singular integrals and differentiability properties of functions, Princeton, Univ. Press, Princeton, N.J., 1970.

10. R. S. Strichartz, A note on Trudinger's extension of Sobolev's inequalities, Indiana Univ. Math. J. 21 (1972), 841-842.

11. N. S. Trudinger, On imbeddings into Orlicz spaces and some applications, J. Math. Mech. 17 (1967), 473-483. MR 35 \#7121.

Department of Mathematics, Uppsala University, Sysslomansgatan 8, UppSALA, SWEDEN 